\title{
Systematic derivation of a rotationally covariant extension of the 2-dimensional Newell-Whitehead-Segel equation
}

\author{
Robert Graham \\ Fachbereich Physik, Universität-Gesamthochschule Essen \\ 45117 Essen \\ Germany
}

\begin{abstract}
An extension of the Newell-Whitehead-Segel amplitude equation covariant under abritrary rotations is derived systematically by the renormalization group method.
\end{abstract}

Typeset using REVTEX 
The present day theory of pattern formation to a large degree rests on the derivation and exploitation of amplitude equations. In particular, for the formation of 2-dimensional patterns by spontaneous symmetry breaking at a finite wavenumber in isotropic 2-dimensional layers the amplitude equation due to Newell and Whitehead and to Segel [1] is of fundamental importance. It is derived, e.g. for the Benard instability, close to the onset of the pattern, by assuming the presence of an ideal one-dimensional roll pattern and certain scaling properties of its small amplitude and its spatial and temporal variations. The small parameter is the difference of the bifurcation parameter from its value at onset.

The resulting amplitude equation, despite of its great fundamental and practical importance, has a well known short-coming: it does not respect the full rotation invariance of the two-dimensional system and the fact, that in principle the rotational symmetry is broken by the pattern spontaneously rather than by any external agent. This short-coming is a direct consequence of the method of derivation, which singles out a particular direction for the main pattern, and even more importantly, makes use of anisotropic assumptions for the scaling of the spatial variation of the pattern.

Two recent developments now permit to overcome this problem [2]. First, Gunaratne et al [3] recently proposed an extended form of the Newell-Whitehead-Segel (N-W-S) equation which they demonstrated to fully respect the rotational symmetry of the original system. They also provided a derivation of their equation by including, in a given order in the bifurcation parameter, symmetry restoring terms, which, indeed appear in higher order of the N-W-S scheme. While this recombining of terms from different orders of the expansion suggests that their equation is the correct symmetric extension of the N-W-S equation, before one can accept this as a fact, a fully systematic (if not mathematically rigorous) derivation 
of this equation must be provided. Clearly, a new method, avoiding any anisotropic scaling assumptions, is required. Recently, such a method has become available with the renormalization group method by Chen et al [4.5], applied to the derivation of amplitude equations [5.6]. Here we shall employ this method to give a systemtic derivation of the rotationally symmetric extension of the N-W-S equation. Curiously, Chen et al [5] and also Kunihiro [6] already used this method to rederive the N-W-S equation, seemingly without invoking the anisotropic scaling assumption of the original derivation. However, as we shall see, such scaling assumptions in fact crept inadvertently into their derivation by the assignement which out of a number of space-time secular terms would be 'most singular'. Avoiding the scaling assumption implicit in their choice and simply keeping all singular terms, but otherwise following closely the treatment in [5] we are able to achieve our goal.

For concreteness and simplicity, we shall present the derivation starting from the twodimensional Swift-Hohenberg equation [0]

$$
\frac{\partial \psi}{\partial t}=\epsilon\left(\psi-\psi^{3}\right)-\left(\frac{\partial^{2}}{\partial x^{2}}+\frac{\partial^{2}}{\partial y^{2}}+k^{2}\right)^{2} \psi
$$

where $\epsilon$ is the bifurcation parameter, and $\psi$ was scaled to make the $\psi$ and $\psi^{3}$-terms both of the same order in $\epsilon$.

The derivation starts with the ansatz

$$
\psi=2 \operatorname{Re}\left\{A e^{i k x}+\epsilon \psi_{1}(x, y)+\cdots\right\}
$$

with amplitude $A$ which is constant to lowest order in $\epsilon$. The ansatz (2) breaks the rotational symmetry in the $(x, y)$-plane. However, due to the rotationally symmetric form of eq. (1) the resulting amplitude equation for $A$ will actually be covariant under rotations of $\boldsymbol{k}=k \hat{e_{x}}$ in eq. (2) in a sense to be made concrete below. Inserting eq. (2) in eq. (1) one obtains an 
equation

$$
\left(\sum_{i=1}^{7} L_{i}\right) \psi_{1}=\left(1-3|A|^{2}\right) A e^{i k x}+\text { non-resonant terms }
$$

with

$$
\begin{aligned}
& L_{1}=\frac{\partial}{\partial t}, L_{2}=\left(\frac{\partial}{\partial x}-i k\right)^{4}, L_{3}=4 i k\left(\frac{\partial}{\partial x}-i k\right)^{3} \\
& L_{4}=-4 k^{2}\left(\frac{\partial}{\partial x}-i k\right)^{2}, L_{5}=2\left(\frac{\partial}{\partial x}-i k\right)^{2} \frac{\partial^{2}}{\partial y^{2}} \\
& L_{6}=4 i k\left(\frac{\partial}{\partial x}-i k\right) \frac{\partial^{2}}{\partial y^{2}}, L_{7}=\frac{\partial^{4}}{\partial y^{4}}
\end{aligned}
$$

The $L_{i}$ turn out all to commute. Now, the space-time secular part of a general solution of eq. (3) is constructed in the form

$$
\begin{aligned}
\psi=2 \operatorname{Re}\left\{A e^{i k x}+\epsilon\left(1-3|A|^{2}\right) A e^{i k x}\right. & \left(C_{1} t+C_{2} \frac{x^{4}}{24}+C_{3} \frac{x^{3}}{24 i k}+C_{4} \frac{-x^{2}}{8 k^{2}}\right. \\
& \left.\left.+C_{5} \frac{x^{2} y^{2}}{8}+C_{6} \frac{x y^{2}}{8 i k}+C_{7} \frac{y^{4}}{24}\right)+\cdots\right\}
\end{aligned}
$$

where we have displayed all secular terms to order $\epsilon$ and the dots denote non-secular or higher order terms. The $C_{i}$ are integration constants and satisfy $\sum_{i} C_{i}=1$. Incidentally, in [5] and [6] the terms $\sim t, x^{2}, x y^{2}, y^{4}$ were considered as 'most singular' and only those terms were kept there. Because this assignment implies different scalings for the other terms and breaks the rotational symmetry we shall not use it here. This accounts for the differences of our results below from those in [5.6].

The renormalization group method [5] now proceeds by (i) introducing an arbitrary 'regularization point' $X, Y, T$ and to reorganize eq. (4) as an expansion around this point, rather than the origin; (ii) splitting in the amplitudes of the secular terms $x^{n}=\left(x^{n}-X^{n}\right)+$ $X^{n}$ and similar for $y^{n}, t^{n}$ where $n$ is any of the exponents of $x, y, t$ appearing there; (iii) absorbing $X^{n}, Y^{n}, T^{n}$, by a redefinition of the amplitude $A$ which thereby becomes a function 
$A(X, Y, T)$. The equation we are left with is eq. (4) with the replacements $A \rightarrow A(X, Y, T)$, $t \rightarrow t-T, x^{n} \rightarrow x^{n}-X^{n}, y^{n} \rightarrow y^{n}-Y^{n}$, but $e^{i k x} \rightarrow e^{i k x}$.

The symmetry which gives rise to the renormalization group is the fact that $\psi$ is independent of $X, Y, T$. Therefore, all derivatives of $\psi$ with respect $X, Y, T$ must vanish. To first order in $\epsilon$ one obtains the conditions

$$
\begin{aligned}
\frac{\partial A}{\partial T}-\epsilon C_{1} A\left(1-3|A|^{2}\right) & =0 \\
\frac{\partial^{4} A}{\partial X^{4}}-\epsilon C_{2} A\left(1-3|A|^{2}\right) & =0 \\
4 i k \frac{\partial^{3} A}{\partial X^{3}}-\epsilon C_{3} A\left(1-3|A|^{2}\right) & =0 \\
-4 k^{2} \frac{\partial^{2} A}{\partial X^{2}}-\epsilon C_{4} A\left(1-3|A|^{2}\right) & =0 \\
2 \frac{\partial^{4} A}{\partial X^{2} \partial Y^{2}}-\epsilon C_{5} A\left(1-3|A|^{2}\right) & =0 \\
4 i k \frac{\partial^{3} A}{\partial X \partial Y^{2}}-\epsilon C_{6} A\left(1-3|A|^{2}\right) & =0 \\
\frac{\partial^{4} A}{\partial Y^{4}}-\epsilon C_{7} A\left(1-3|A|^{2}\right) & =0
\end{aligned}
$$

Adding these equations by using $\sum_{i} C_{i}=1$ and identifying $X, Y, T \rightarrow x, y, t$ one obtains $\psi=2 \operatorname{Re}\left\{A(x, y, z, t) e^{i k x}\right\}$ instead of (四) and the amplitude equation, independent of the $C_{i}$, to order $\epsilon$

$$
\frac{\partial A}{\partial t}-\epsilon A\left(1-3|A|^{2}\right)=4 k^{2} \square_{x}^{2} A
$$

with the symbol $\square_{x}$ introduced in [3] as

$$
\square_{x}=\hat{\boldsymbol{e}}_{x} \cdot \nabla-\frac{i}{2 k} \nabla^{2}
$$

The N-W-S equation is also of the form (5), but there $\square_{x}^{2}$ is replaced by $\left(\frac{\partial}{\partial x}-\frac{i}{2 k} \frac{\partial^{2}}{\partial y^{2}}\right)^{2}$. It is obtained for special solutions of (3) with $C_{2}=C_{3}=C_{5}=0$. This completes our derivation. 
It is clear that the method is systematic and may be extended to higher order in $\epsilon$. It can be seen that the particular form $\square_{x}$ of the spatial derivative operator is a direct consequence of the form of all secular terms in (田), which are in turn a consequence of the rotational invariance of the derivative operator in (11). Hence $\square_{x}$ will automatically be generated by this method starting from any rotationally invariant set of equations in place of (回), like e.g. the Boussinesq equations for thermal convection.

The covariance of eq. (5) under rotations has been discussed in [3]. It rests on the remarkable property $\square_{x} e^{i \Delta \boldsymbol{k}_{x} \boldsymbol{x}}=0$ [3], where $\Delta \boldsymbol{k}_{x}=\boldsymbol{k}(\vartheta)-k \hat{\boldsymbol{e}}_{x}=k\left((\cos \vartheta-1) \hat{\boldsymbol{e}}_{x}+\sin \vartheta \hat{\boldsymbol{e}}_{y}\right)$, and the even more remarkable property

$$
e^{-i \Delta \boldsymbol{k}_{x} \boldsymbol{x}} \square_{x} e^{i \Delta \boldsymbol{k}_{x} \boldsymbol{x}}=\square_{x^{\prime}},
$$

where $\hat{\boldsymbol{e}}_{x^{\prime}}=\cos \vartheta \hat{\boldsymbol{e}}_{x}+\sin \vartheta \hat{\boldsymbol{e}}_{y}$. It is the second property (which is independent of the first because $\square_{x}$ is of second order) which makes in eq. (5) the transformation of the amplitude $A \rightarrow A e^{i \Delta \boldsymbol{k}_{x} \cdot \boldsymbol{x}}$ equivalent to the rotation $k \hat{\boldsymbol{e}}_{x} \rightarrow k \hat{\boldsymbol{e}}_{x^{\prime}}$ of the wave-vector.

As many properties of patterns like e.g. defects and textures depend in a crucial manner on the underlying symmetry and the fact that it is spontaneously, not externally, broken, the covariance of eq. (5) under rotations must be considered an important improvement of the fundamental N-W-S equation. In addition we may remark that the derivation we have given would still apply if noise is added on the right hand side of (1) providing a rotationally covariant extension of the noisy amplitude equation derived in [8].

Like the N-W-S equation the amplitude equation (5) has a potential, i.e. it can be written in the form $\frac{\partial A}{\partial t}=-\frac{\delta \phi}{\delta A^{*}}$ with the Lyapunov functional

$$
\phi=\int_{d x d y}\left\{-\epsilon|A|^{2}\left(1-\frac{3}{2}|A|^{2}\right)+4 k^{2}\left|\square_{x} A\right|^{2}\right\} .
$$


However, unlike the potential for the N-W-S equation [8] (where again $\square_{x}$ is replaced by $\left.\left(\frac{\partial}{\partial x}-\frac{i}{2 k} \frac{\partial^{2}}{\partial y^{2}}\right)\right)$ the potential (6) has minima, the attractors of eq. (5), for $A=(3)^{-1 / 2} e^{i \Delta \mathbf{k}(\vartheta) \cdot \mathbf{x}}$ with arbitrary angle $\vartheta$. In the noisy case $\mathrm{W}\left(\left\{A, A^{*}\right\}\right)=N \exp [-\phi / Q]$ is the steady-state distribution of the fluctuating amplitude. Here $Q$ is a measure of the noise intensity . For the case of Bénard convection at finite temperature it has been calculated in [8]. Again rolls in arbitrary directions in the $(x, y)$-plane appear with equal weight in this distribution, in contrast to the case of the $\mathrm{N}$-W-S equation where $\boldsymbol{k}$-vectors parallel to the $x$-axis are strongly preferred [8].

In summary, we have given a systematic derivation of the amplitude equation for the formation of roll patterns in 2-dimensional systems by spontaneous symmetry breaking. The derivation is free from anisotropic scaling assumptions and establishes a rotationally forminvariant extension of the N-W-S equation as the fundamental amplitude equation for such systems.

\section{Acknowledgements}

This work was supported by the Deutsche Forschungsgemeinschaft through its Sonderforschungsbereich 237 "Unordnung und große Fluktuationen". 


\section{REFERENCES}

[1] A. C. Newell and J. A. Whitehead, J. Fluid Mech. 38, 279 (1969); L. A. Segel, J. Fluid Mech. 38, 203 (1969)

[2] Another approach to overcome the spurious breaking of rotational symmetry complementary to the present one (see [3] for a comparison), is due to M.C. Cross and A.C. Newell, Physica D10, 299 (1984); A.C. Newell, T. Passot and J. Lega, Ann. Rev. Fluid Mech. 25, $399(1993)$

[3] G. H. Gunaratne, Qi Ouyang, and H. L. Swinney, Phys. Rev. E50, 2802 (1994)

[4] L. Y. Chen, N. Goldenfeld, Y. Oono and G. Paquette, Physica A204, 111 (1994); G. Paquette, L. Y. Chen, N. Goldenfeld and Y. Oono, Phys. Rev. Lett. 72, 76 (1994); L. Y. Chen, N. Goldenfeld and Y. Oono, Phys. Rev. Lett 73,1311 (1994)

[5] L. Y. Chen, N. Goldenfeld and Y. Oono, preprint 1995, hep-th/9506161;

[6] T. Kunihiro, preprint 1995, RYUTHP-95/4

[7] J. Swift and P. C. Hohenberg, Phys. Rev. A15, 319 (1977)

[8] R. Graham, Phys. Rev. Lett. 31, 1479 (1973); Phys. Rev. A10, 1762 (1974); A45, 4198(E) (1992) 